\title{
Interaction between early environment and genetic predisposition instigates the metabolically obese, normal weight phenotype in children: findings from the BCAMS study
}


Jie $\mathbf{M i}^{5}$ and Shan Gao ${ }^{2}$

'Department of Endocrinology, NHC Key Laboratory of Endocrinology, Peking Union Medical College Hospital, Peking Union Medical College and Chinese Academy of Medical Sciences, Beijing, China, ${ }^{2}$ Department of Endocrinology, Beijing Chaoyang Hospital, Capital Medical University, Beijing, China, ${ }^{3}$ Department of Epidemiology, School of Public Health and Tropical Medicine, Tulane University, New Orleans, Louisiana, USA, ${ }^{4}$ Department of Endocrinology, The Children's Hospital of Philadelphia, Perelman School of Medicine, University of Pennsylvania, Philadelphia, Pennsylvania, USA,

${ }^{5}$ Department of Epidemiology, Capital Institute of Paediatrics, Beijing, China, and ${ }^{6}$ Department of Nutrition, Harvard T.H. Chan School of Public Health, Boston, Massachusetts, USA

Correspondence should be addressed to $\mathrm{M} \mathrm{Li}$ Email liming@pumch.cn

\begin{abstract}
Objective: A subset of normal-weight individuals appears predisposed to obesity-related cardiometabolic abnormalities. Studies of this metabolically obese, normal weight (MONW) phenotype in youth are scarce. We aimed to identify early environmental and genetic factors associated with MONW in children.

Methods: Overall, 1475 normal-weight Chinese children aged 6-18 were recruited from the Beijing Children and Adolescents Metabolic Syndrome study cohort. Birthweight, childhood lifestyle, socio-economic factors, and 20 genetic variants previously shown to be associated with BMI or glucose metabolism in East Asian adults were examined for their association with the MONW phenotype. MONW was defined by exhibiting any metabolic syndrome component. Results: After adjusting for covariates including BMI, low birthweight and low levels of physical activity, fruit consumption, parental education and household income, as well as CDKAL1 rs2206734 genotype were independent predictors of the MONW phenotype (all $P<0.05$ ). Moreover, rs2206734 interacted with birthweight to predict the MONW phenotype $\left(P_{\text {interaction }}=0.0008\right)$. Among high $(>75$ th percentile) birthweight individuals, each $\mathrm{C}$ allele at this locus was associated with a $62 \%$ reduced risk of MONW $\left(\mathrm{OR}=0.38 ; 95 \% \mathrm{Cl}=0.26-0.58 ; P=5.71 \times 10^{-6}\right)$, while no such genetic associations were found in intermediate or low birthweight individuals $(P>0.1)$. This CDKAL1-MONW relationship in high birthweight individuals was especially strong in the presence of favorable childhood environmental factors (high levels of physical activity, fruit consumption, parental education and household income) $\left(P_{\text {interaction }}=0.013\right)$. Conclusions: Our findings provided the novel evidence that early environment (especially birthweight) and genetics, along with their interaction with one another, play important roles in predicting the MONW phenotype among children.
\end{abstract}

European Journal of Endocrinology (2020) 182, 393-403 


\section{Introduction}

Obesity is a well-known cardiometabolic risk factor. However, some individuals, despite a healthy BMI, are at increased risk of cardiometabolic disorders, including type 2 diabetes (T2D), hypertension and cardiovascular disease (CVD). These people are referred to as 'metabolically obese, normal weight' (MONW) (1). Both genetic and environmental factors are known to contribute to the development of the MONW phenotype (1); however, unlike metabolic defects in subjects with obesity, the factors underlying the pathogenesis in normal-weight individuals are largely unknown. Previous studies have shown that Asians are at greater risk for cardiometabolic abnormalities compared to their Western counterparts, even at relatively low BMI levels $(2,3)$. Yet, the factors underlying this increased CVD risk remain unclear. Because children exhibit metabolic plasticity, and are relatively free of co-morbidities and confounding treatment effects, they represent an ideal population in which to study the pathogenesis of MONW. Nevertheless, we can find no studies which assess environmental and genetic factors contributing to the MONW phenotype in Asian children.

While the pathogenesis of MONW remains unclear, recent evidence suggests that adipose tissue dysregulation may contribute via the 'adipose expandability' hypotheses, which states that inadequate s.c. adipose tissue expansion in response to ingested triglycerides may lead to visceral and ectopic fat deposition with inherent insulin resistance and cardiometabolic dysfunction, even in the face of normal body weight $(1,4,5)$. A genetic basis for MONW can be inferred from the myriad of genome-wide association studies (GWAS) which identify various genetic variants associated with fat mass or body fat distribution, and which involve pathways relevant to adipose tissue dysregulation $(4,6)$. In addition, a number of genetic variants from GWAS have been associated with carbohydrate and lipid metabolism disorders and are worthy of investigation for their ability to confer a predisposition to $\operatorname{MONW}(7,8)$.

Increasing evidence suggests that childhood environmental exposures, such as lack of physical activity, unfavorable dietary patterns and low socioeconomic status, play a significant role in metabolic health $(9,10$, 11). However, no study has systematically assessed the individual and combined effects of environmental factors in early-life or the interaction between environmental patterns and genetic susceptibility in determining the pediatric MONW phenotype. While it is well established that the intrauterine environment has a persistent impact on metabolism and gene expression in later life (12), relevant studies examining the early origins of the MONW phenotype are scarce.

Leveraging the large dataset from the Beijing Children and Adolescents Metabolic Syndrome (BCAMS) study, we aimed to explore the contribution of genetic and environmental factors to the pathogenesis of the MONW phenotypein childhood. In addition, weexamined whether individual or a multiplex combination of environmental factors during critical periods of development can modify the genetic predisposition to the MONW phenotype. Our study may shed light on underlying mechanisms and help distinguish between those children who are most and least prone to developing cardiometabolic impairment despite their body weight being within the normal range, and identify a path toward optimization of early prevention and treatment strategies.

\section{Subjects and methods}

\section{Subjects}

Study participants were from the BCAMS study, which is a longitudinal study of cardiometabolic risk factors from childhood to young adulthood in a representative sample of school-age children $(n=19,593$, aged $6-18$ years, 50\% boys) from Beijing $(13,14)$. The baseline survey began in 2004 and has been described in detail elsewhere (14). Within this cohort, 4500 participants were recognized as having risk factors defined by the presence of at least one of the following: overweight (defined as $\mathrm{BMI}>85$ th percentile), total cholesterol (TC) $\geq 5.2 \mathrm{mmol} / \mathrm{L}$, triglyceride $(\mathrm{TG}) \geq 1.7 \mathrm{mmol} / \mathrm{L}$ or fasting blood glucose $\geq 5.6 \mathrm{mmol} / \mathrm{L}$ based on capillary blood tests. Along with a parallel reference sample of 1095 children, these participants were invited to undergo further medical examinations for verification based on venipuncture blood samples and a clinical examination. Among these cohorts, 3235 children completed further medical examination and provided complete data, including information about lifestyles, family history, and parental education. In the current analysis, we focused on the 1475 normal-weight individuals (594 boys) from the 3235 children with complete data (Supplementary Fig. 1, see section on supplementary materials given at the end of this article). Children with a known history of type 1 or type 2 diabetes were excluded, and normal weight was defined by a sex- and age-specific BMI between the 5 th 
and 85 th percentiles. The study protocol was approved by the Ethics Committee at the Capital Institute of Pediatrics and Peking Union Medical College Hospital. Signed informed consent was obtained from all participants and/ or their parents or guardians. The study protocol was also registered at www.clinicaltrials.gov (NCT03421444).

\section{Anthropometric and biochemical parameters}

Weight, height, waist circumference (WC), blood pressure and fat mass percentage (FAT\%) by bio-impedance analysis were measured by trained staff as previously reported (14, 15). Pubertal development was scored by Tanner stage for breast development in girls and testicular volume in boys. This assessment was performed by two pediatricians of the same gender as the child. Venous blood samples were collected after an overnight $(\geq 12 \mathrm{~h})$ fast. Fasting plasma concentrations of glucose (FPG), TG, TC, HDL cholesterol (HDL-C), and LDL cholesterol (LDL-C) were assayed by a Hitachi $7060 \mathrm{C}$ automatic biochemistry analysis system. Fasting insulin was measured by an ELISA developed at the Key Laboratory of Endocrinology, Peking Union Medical College Hospital, without cross-reactivity to proinsulin $(<0.05 \%)(13,14)$. Homeostasis model assessment of insulin resistance (HOMA-IR) was calculated as fasting insulin $(\mathrm{mU} / \mathrm{L}) \times \mathrm{FPG}(\mathrm{mmol} / \mathrm{L}) / 22.5$ (16).

\section{Assessment of environmental factors}

The intrauterine environment was assessed from birthweight, with $<25$ th, 25th-75th and $>75$ th percentiles equated with low, intermediate and high birthweights, respectively. Questionnaires were used to obtain information on lifestyle factors including diet, physical activity, sleep duration, and mode of transportation to school as previously described $(13,15)$. Dietary factors were assessed with a food frequency intake assessment of ten items that were dichotomized according to their median frequency. Physical activity was assessed by the frequency of vigorous exercise including cycling, hiking, batting, running, swimming and other extracurricular physical activities performed for at least $30 \mathrm{~min}$ in duration and was classified as low ( $<3$ times/week) or high ( $\geq 3$ times/week) levels of moderate-to-vigorous physical activity (MVPA). Additionally, the mode of transportation to school was divided into two categories as 'walking' and 'nonwalking'. Sleep duration was recorded as the response to 'How many hours of sleep do you usually get at night?' Socioeconomic status was assessed by maximum parental education level attained and annual household income (13). Highest level of parental education was classified as $<$ University or $\geq$ University. Annual household income was categorized in quartiles as low $(<15,600$ yuan, $<25$ th percentiles), moderate (15,600-58,000 yuan, 25th-75th percentiles), or high ( $>58,000$ yuan, $>75$ th percentiles).

In order to combine the multiplex of childhood environmental factors into a single element for analysis, we derived a composite score by enumerating the independent protective environmental factors. Every independent protective environmental factor including MVPA, high fruit consumption, parental education $\geq$ university, or high annual household income was assigned a score of 1 , while low physical activity, low fruit consumption, parental education $<$ university, low or moderate annual household income was ranked as a 0 , according to their presumed effects on metabolic health. Furthermore, low and high composite scores were defined as $0-1$ and $2-4$ protective factors, respectively.

\section{SNP selection and genotyping}

Genomic DNA was extracted from blood samples using the QIAamp DNA Blood Midi Kits. We selected 20 SNPs including BMI-related loci (13) and glucose metabolismrelated loci (7) previously reported in GWAS studies of East Asian populations (Supplementary Table 1). Genotyping was performed on the Sequenom Mass Array iPLEX genotyping platform in BioMiao Biological Technology (Beijing) Co, Ltd. (17). Repeated control samples were present in each genotyping plate with the concordance rate being $100 \%$. All SNPs had a genotyping efficiency of greater than 95\%, but two SNPs were excluded due to deviation from Hardy-Weinberg equilibrium with a Bonferroni-adjusted significance level of $P<0.0025$ (0.05 divided by 20).

\section{Definitions of metabolically obese, normal weight}

The MONW phenotype was defined by the presence of any classic component of the pediatric metabolic syndrome (MS) including (14): (1) hypertension (SBP and/ or $\mathrm{DBP} \geq 90$ th percentile for age, sex and height according to BCAMS); (2) hypertriglyceridemia (TG $\geq 1.24 \mathrm{mmol} / \mathrm{L}$ ); (3) low serum HDL-C (defined as $\leq 1.03 \mathrm{mmol} / \mathrm{L}$ ), or (4) impaired FPG (defined as $\geq 5.6 \mathrm{mmol} / \mathrm{L}$ ), occurring in a normal-weight child. The metabolically healthy normalweight (MHNW) phenotype was defined by the absence of any such features of MS. 


\section{Statistical analysis}

Any parameters whose values were not normally distributed underwent logarithmic transformation before analysis. All results are expressed as mean \pm S.D., unless otherwise stated. Group differences of continuous or categorical variables in children with or without the MONW phenotype were evaluated by independent sample $t$ tests or $\chi^{2}$ tests, respectively. The associations between individual SNPs and MONW were tested using additive models. Each SNP was recoded as 0,1 , or 2 according to the number of high risk alleles. Logistic regression analysis was used to assess the associations between each of the variables (including SNPs) and the MONW phenotype. Variables found to be significant in the age-, gender-, puberty- and BMIadjusted analyses were added to a multivariable logistic regression model to evaluate the independent risk factors of MONW. Moreover, interactions between individual SNPs and individual environmental factors (or combined factor patterns) on MONW were examined using logistic regression analysis by adding the relevant interaction terms into the models. For example, birthweight $\times \mathrm{SNP}$, with inclusion of the main effects in the models. Power calculation was performed by Quanto software version 1.2.3 (University of Southern California, Los Angeles, CA, USA). All statistical analyses were performed using SPSS version 22.0 software for windows (SPSS Inc.).

\section{Results}

\section{Subject characteristics according to metabolic health status in normal weight children}

Table 1 shows the characteristics of normal-weight subjects with or without metabolic abnormalities. There were $531(36.0 \%)$ children defined as MONW. Factors associated with the MONW phenotype after adjusting for age, gender, pubertal status and BMI are shown in Table 2. Increased FAT\% was related to MONW $(P<0.001)$. Among the lifestyle factors evaluated, consumption of bean, meat, seafood and fruit, as well as increased physical activity (whether from walking to school or extracurricular MVPA) were associated with a reduced risk of MONW after adjustment for covariates (all $P<0.05$ ). Furthermore, a lower birthweight was linked to greater risk of MONW $(P<0.05)$. Finally, lower levels of parental education and household income were associated with the MONW phenotype (all $P<0.05$ ). The statistics for all genetic variants analyzed are listed in Supplementary
Table 1. Only CDKAL1-rs2206734 showed a significant association with the MONW phenotype after Bonferroni correction $(P=0.002)$.

\section{Independent predictors of the MONW phenotype}

To identify the independent factors related to metabolic aberration in normal weight individuals, all the significant variables in Table 2 were included in the final models for multivariate analysis. Regarding the intrauterine environment, participants with the highest birthweight ( $>75$ th percentile) had the lowest risk of MONW, independent of childhood BMI (Table 3). Compared to less physically active individuals, children with greater amounts of exercise had a significant reduction $(26 \%)$ in the likelihood of exhibiting metabolically obesity (95\% $\mathrm{CI}=0.58-0.93, P=0.011$ ). Higher consumption of fruit was also a protective factor for MONW $(\mathrm{OR}=0.75,95 \%$ $\mathrm{CI}=0.59-0.95, P=0.018$ ). Analysis of the contribution of combined childhood environmental factors revealed that the composite score (which involves a combination of protective factors including physical activity, fruit consumption, parental education and annual household income) was strongly related to the MONW phenotype with an odds ratio (OR) per factor increase of 0.68 (95\% $\mathrm{CI}=0.62-0.76, P<0.001)$. As for genetic associations, after controlling for the aforementioned risk variables, only the CDKAL1-rs2206734 genotype showed an association with protection from the MONW phenotype, with each additional C allele in rs2206734 having a 21\% decrease in the odds of exhibiting MONW (95\% CI=0.67-0.93, $P=0.005)$.

\section{Gene-environment interaction effects on the MONW phenotype}

Since the CDKAL1 rs2206734 locus had the strongest association with the MONW phenotype, we further examined the association between this variant and the environmental factors influencing the risk of MONW, and no significant association was evident in our study population (data not shown). We therefore examined whether the significant environmental factors listed in Table 3 could modify the effect of this key genetic variant on MONW risk by testing for their interactions. As shown in Fig. 1A, a significant interaction between the CDKAL1 locus and birthweight was observed in determining the MONW phenotype, even after adjusting for age, gender, pubertal status, gestational age, BMI, physical activity level, fruit consumption, parental education, and household 
Table 1 Comparison of characteristics between MONW and MHNW. Data are shown as the mean \pm S.D. or median (interquartile range) or percentage.

\begin{tabular}{|c|c|c|c|}
\hline Variables & MHNW & MONW & $P$ value \\
\hline \multicolumn{4}{|l|}{ Demography } \\
\hline$n$ & 944 & 531 & \\
\hline Age (years) & $12.2 \pm 3.1$ & $12.9 \pm 3.1$ & $<0.001$ \\
\hline Male $(\%)$ & 41.6 & 37.9 & 0.156 \\
\hline Tanner stage (\%) & & & $<0.001$ \\
\hline 1 & 30.9 & 21.5 & \\
\hline 2 & 15.8 & 15.7 & \\
\hline 3 & 14.8 & 13.8 & \\
\hline 4 & 28.4 & 33.7 & \\
\hline 5 & 10.1 & 15.3 & \\
\hline BMI Z-score & $-0.31 \pm 0.86$ & $-0.14 \pm 0.81$ & $<0.001$ \\
\hline $\mathrm{BMI}\left(\mathrm{kg} / \mathrm{m}^{2}\right)$ & $17.54 \pm 2.39$ & $18.26 \pm 2.45$ & $<0.001$ \\
\hline WC $(\mathrm{cm})$ & $61.67 \pm 7.09$ & $63.25 \pm 7.06$ & $<0.001$ \\
\hline FAT\% & $17.48 \pm 5.49$ & $19.21 \pm 5.87$ & $<0.001$ \\
\hline \multicolumn{4}{|l|}{ Family history } \\
\hline Father, diabetes (\%) & 1.3 & 2.1 & 0.234 \\
\hline Mother, diabetes (\%) & 0.8 & 0.8 & 0.847 \\
\hline Father, hypertension (\%) & 6.7 & 7.5 & 0.534 \\
\hline Mother, hypertension (\%) & 2.8 & 4.0 & 0.208 \\
\hline Father, dyslipidemia (\%) & 4.8 & 4.5 & 0.829 \\
\hline Mother, dyslipidemia (\%) & 1.3 & 1.3 & 0.939 \\
\hline Birthweight (kg) & $3.35 \pm 0.53$ & $3.22 \pm 0.47$ & $<0.001$ \\
\hline Gestational age (\%) & & & 0.264 \\
\hline Premature & 5.4 & 5.9 & \\
\hline Full-term & 90.8 & 88.5 & \\
\hline Overdue & 3.8 & 5.7 & \\
\hline \multicolumn{4}{|l|}{ Lifestyle factors } \\
\hline Breakfast $\geq 5$ times/week (\%) & 81.0 & 76.8 & 0.059 \\
\hline Bean $\geq 3$ times/week (\%) & 37.4 & 27.7 & $<0.001$ \\
\hline Meat $\geq 5$ times/week (\%) & 51.8 & 37.5 & $<0.001$ \\
\hline Seafood $\geq 1$ time/week (\%) & 21.1 & 16.0 & 0.017 \\
\hline Dairy $\geq 5$ times/week (\%) & 59.3 & 42.9 & $<0.001$ \\
\hline Vegetable $\geq 5$ times/week (\%) & 89.7 & 86.6 & 0.075 \\
\hline Fruit $\geq 5$ times/week (\%) & 63.7 & 50.7 & $<0.001$ \\
\hline Fried food $\geq 1$ time/week (\%) & 33.4 & 33.6 & 0.931 \\
\hline Soft drink $\geq 1$ time/week (\%) & 45.1 & 42.1 & 0.284 \\
\hline Fast food $\geq 1$ time/week (\%) & 13.8 & 10.0 & 0.035 \\
\hline MVPA (\%) & 63.1 & 53.7 & $<0.001$ \\
\hline Walk to school (\%) & 69.0 & 56.2 & $<0.001$ \\
\hline Sleep duration (hours/day) & $8.59 \pm 1.24$ & $8.52 \pm 1.22$ & 0.336 \\
\hline \multicolumn{4}{|l|}{ Socioeconomic factors } \\
\hline Parental education $\geq$ University (\%) & 33.5 & 17.9 & $<0.001$ \\
\hline Annual household income & & & $<0.001$ \\
\hline Low (<25th percentiles) & 20.4 & 35.8 & \\
\hline Intermediate (25th-75th percentiles) & 46.6 & 46.9 & \\
\hline High ( $>75$ th percentiles) & 32.9 & 17.3 & \\
\hline \multicolumn{4}{|l|}{ Cardiometabolic risk } \\
\hline $\mathrm{SBP}(\mathrm{mmHg})$ & $97.9 \pm 10.5$ & $107.9 \pm 12.8$ & $<0.001$ \\
\hline $\mathrm{DBP}(\mathrm{mmHg})$ & $61.6 \pm 8.0$ & $69.0 \pm 10.3$ & $<0.001$ \\
\hline Total cholesterol (mmol/liter) & $4.10 \pm 0.90$ & $4.17 \pm 0.93$ & 0.117 \\
\hline Triglycerides (mmol/liter)* & $0.71(0.56-0.90)$ & $0.98(0.69-1.44)$ & $<0.001$ \\
\hline HDL-C (mmol/liter) & $1.58 \pm 0.29$ & $1.45 \pm 0.34$ & $<0.001$ \\
\hline LDL-C (mmol/liter) & $2.44 \pm 0.73$ & $2.57 \pm 0.87$ & 0.020 \\
\hline FPG (mmol/liter) & $4.88 \pm 0.38$ & $5.23 \pm 0.53$ & $<0.001$ \\
\hline Insulin $(\mathrm{mU} / \mathrm{L})^{*}$ & $5.09(3.14-7.74)$ & $7.16(4.88-10.02)$ & $<0.001$ \\
\hline HOMA-IR* & $1.12(0.65-1.73)$ & $1.65(1.11-2.40)$ & $<0.001$ \\
\hline
\end{tabular}


Table 2 Associations between lifestyle and socioeconomic factors, genetic predisposition and metabolically obese, normal weight.

\begin{tabular}{l}
\hline \\
\hline Anthropometry \\
WC (cm) \\
FAT\% \\
Birthweight (kg) \\
Intermediate (25th-75th percentiles) \\
$\quad$ Low ( $<25$ th percentiles) \\
High ( $>75$ th percentiles) \\
Lifestyle factors \\
Breakfast $\geq 5$ times/week (\%) \\
Bean $\geq 3$ times/week (\%) \\
Meat $\geq 5$ times/week (\%) \\
Seafood $\geq 1$ time/week (\%) \\
Vegetable $\geq 5$ times/week (\%) \\
Fruit $\geq 5$ times/week (\%) \\
Soft drink $\geq 1$ time/week (\%) \\
Fast food $\geq 1$ time/two week (\%) \\
MVPA (\%) \\
Walk to school (\%) \\
Sleep duration (h/day) \\
Socioeconomic factors \\
Parental education $\geq$ University \\
Annual household income \\
Low ( $<25$ th percentiles) \\
Intermediate (25th-75th percentiles) \\
High ( $>75$ th percentiles) \\
SNP \\
CDKAL1-rs2206734-C* \\
\hline
\end{tabular}

\begin{tabular}{c}
\hline OR \\
\hline 0.97 \\
1.04 \\
0.55 \\
1 \\
1.38 \\
0.64 \\
\\
0.86 \\
0.65 \\
0.55 \\
0.73 \\
0.74 \\
0.60 \\
0.83 \\
0.71 \\
0.71 \\
0.60 \\
1.08 \\
\\
0.43 \\
1 \\
0.55 \\
0.30 \\
0.78 \\
\hline
\end{tabular}

\begin{tabular}{c}
\hline $\mathbf{9 5 \%} \mathbf{~ C l}$ \\
\hline $0.93-1.01$ \\
$1.02-1.07$ \\
$0.44-0.70$ \\
- \\
$1.08-1.78$ \\
$0.48-0.86$ \\
$0.66-1.13$ \\
$0.51-0.82$ \\
$0.44-0.68$ \\
$0.55-0.97$ \\
$0.53-1.03$ \\
$0.48-0.75$ \\
$0.66-1.04$ \\
$0.51-1.00$ \\
$0.57-0.88$ \\
$0.48-0.75$ \\
$0.97-1.19$ \\
$0.33-0.56$ \\
- \\
$0.43-0.71$ \\
$0.22-0.41$ \\
$0.66-0.91$ \\
\hline
\end{tabular}

\begin{tabular}{c}
\hline P value \\
\hline 0.091 \\
$<0.001$ \\
$<0.001$ \\
- \\
$\mathbf{0 . 0 1 0}$ \\
$\mathbf{0 . 0 0 3}$ \\
0.284 \\
$<0.001$ \\
$<\mathbf{0 . 0 0 1}$ \\
$\mathbf{0 . 0 2 7}$ \\
0.072 \\
$<\mathbf{0 . 0 0 1}$ \\
0.106 \\
0.053 \\
$\mathbf{0 . 0 0 2}$ \\
$<\mathbf{0 . 0 0 1}$ \\
0.168 \\
$<\mathbf{0 . 0 0 1}$ \\
- \\
$<\mathbf{0 . 0 0 1}$ \\
$<\mathbf{0 . 0 0 1}$ \\
$\mathbf{0 . 0 0 2}$ \\
\hline
\end{tabular}

*Additive genetic model was used. ORs were adjusted for age, gender, puberty and BMI. Boldface type indicates nominally significant values $(P<0.05)$ MHNW, metabolically healthy normal weight; MONW, metabolically obese, normal weight; MVPA, moderate-to-vigorous physical activity; OR, odds ratio; WC, waist circumference.

income ( $P$ for interaction $=0.0008)$. Notably, every $\mathrm{C}$ allele at the CDKAL1 locus reduced the risk of MONW by $62 \%$ in people with high birthweight $(95 \% \mathrm{CI}=0.26-$ $\left.0.58, P=5.71 \times 10^{-6}\right)$ as compared to the (insignificant) associations observed in children of intermediate and low birthweight $(P>0.100)$.

As the genetic effects of CDKAL1 were clearly modified by the intrauterine environment, we explored whether

Table 3 Independent factors of metabolically obese, normal weight.

\begin{tabular}{l}
\hline Variable \\
\hline Birthweight (kg) \\
Low (<25th percentiles) \\
Intermediate (25th percentiles-75th percentiles) \\
High ( $>75$ th percentiles) \\
Parental education $\geq$ University \\
Annual household income \\
Low (<25th percentiles) \\
Intermediate (25th-75th percentiles) \\
High ( $>75$ th percentiles) \\
Fruit $\geq 5$ times/week (\%) \\
MVPA (\%) \\
CDKAL1-rs2206734-C*
\end{tabular}

\begin{tabular}{c}
\hline OR \\
\hline 1 \\
0.80 \\
0.56 \\
0.63
\end{tabular}

\begin{tabular}{ccc}
\hline $\mathbf{9 5 \%} \mathbf{~ C l}$ & & P value \\
$0.61-1.04$ & & - \\
$0.40-0.78$ & & 0.093 \\
$0.47-0.86$ & & $\mathbf{0 . 0 0 1}$ \\
$\mathbf{0 . 0 0 3}$
\end{tabular}

Variables adjusted for age, gender, puberty and BMI and a $P$ value $\leq 0.05$ was selected in the model

*Additive genetic model was used. ORs were adjusted for age, gender, puberty, BMI and gestational age. Boldface type indicates nominally significant values $(P<0.05)$.

OR, odds ratio. 


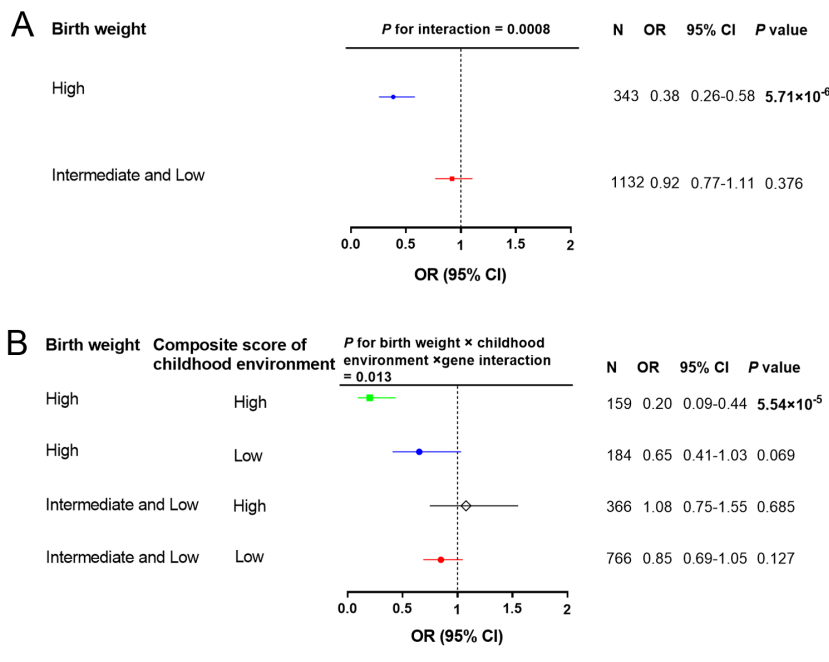

\section{Figure 1}

The effects of the C allele of CDKAL1-rs2206734 in the stratification of birthweight (A) or birth weight and childhood environmental patterns (B) on the metabolically obese, normal weight phenotype. The data are shown as OR $(95 \% \mathrm{Cl})$. The ORs for each C allele of CDKAL1-rs2206734 in the stratification of birthweight were adjusted for age, gender, puberty, gestational age, BMI, physical activity, fruit consumption, parental education and annual household income. The composite score of protective childhood environmental factors was the combination of high physical activity, fruit consumption, parental education and annual household income. The ORs for each C allele in the stratification of birth weight and childhood environmental factors were adjusted for age, gender, puberty, gestational age, and BMI. $P$ values for the gene-environment interaction on the MONW phenotype were adjusted for the above variables.

childhood environmental factors could further modify the impact of intrauterine environment on CDKAL1 effects. A significant interaction between birthweight, childhood environments and the CDKAL1 locus was found ( $P$ for interaction=0.013; Fig. 1B). Within the high birthweight strata, every C allele of the rs2206734 variant was linked to an $80 \%$ reduction in MONW risk in children with favorable childhood environments, while in children with unfavorable childhood environments, every C allele of rs220673 was associated with only a 35\% reduced risk of MONW, a difference of only marginal significance $(\mathrm{OR}$ per $\mathrm{C}$ allele $=0.20,95 \% \mathrm{CI}=0.09-0.44$ vs $\mathrm{OR}=0.65,95 \% \mathrm{CI}=0.41-1.03)$. These findings suggest that a favorable childhood environment can further amplify the protective effect of the CDKAL1 locus in children with high birthweight. Within the strata of intermediate and low birthweight, however, the contribution of $\mathrm{C}$ alleles within rs2206734 was not significant in both favorable and unfavorable childhood environments $(\mathrm{OR}=1.08,95 \%$ $\mathrm{CI}=0.75-1.55$ and $\mathrm{OR}=0.85,95 \% \mathrm{CI}=0.69-1.05)$. This finding suggests that improving childhood environmental factors does not amplify the effect of the CDKAL1 locus on MONW risk in intermediate and low birthweight individuals.

\section{Discussion}

In this cohort of Chinese children, we found that lifestyle and socioeconomic factors, as well as low birthweight, were independent risk factors for the MONW phenotype. In addition, we identified an association in this population between the MONW phenotype and SNPs at the obesity susceptibility locus (CDKAL1) within the gene encoding cyclin dependent kinase 5 (CDK5) regulatory subunit-associated protein 1-like 1 . Moreover, we provide evidence that the protective effects of the CDKAL1-rs2206734 gene variant could be strengthened by a favorable intrauterine nutritional environment and amplified further by the combined impact of protective childhood environmental factors like physical activity, fruit consumption, advanced parental education and high household income. Our study provides the first evidence of gene-environment interactions predisposing to the MONW phenotype, suggesting the importance of identifying genetically susceptible individuals for targeted fetal and childhood environmental modifications to achieve disease prevention.

In this study, we were able to take into account several potential risk factors for MONW based on our large well-characterized cohort of children. We found not only behavioral factors such as physical activity and fruit consumption, but also socioeconomic components including parental education and household income have an independent impact on childhood MONW status. Consistent with our results, a prior pediatric study demonstrated an association between physical activity and metabolic health in children with normal weight and obesity (18). Furthermore, socioeconomic status has been shown to influence food choices, access to health care, and exposure to environmental stressors that directly impact metabolic health $(11,19,20)$, which emphasizes the role of childhood environment as a modifiable risk factor for MONW.

In addition to postnatal environment, low birthweight strongly predicted childhood MONW status in our study. Birthweight is a reflection of the intrauterine 
nutritional environment, which may permanently affect the function of various organ systems, including the endocrine pancreas and adipose tissue, through epigenetic modifications which thereby impact the risk of subsequent development of cardiovascular disease $(21,22)$. Furthermore, our research evinces for the first time that low ( $\leq 25$ th percentile) birthweight contributes to the development of childhood MONW, while high (>75th percentile) birthweight was more likely to impart a protective effect against metabolic disorders in children who subsequently assume a normal weight. It has been reported that children with high birthweight exhibit higher peripheral versus central fat distribution, and higher insulin sensitivity than their normal birthweight peers. This occurs in spite of similar percentages of total body fat mass (23). Meanwhile, low birthweight has been associated with reduced lean tissue mass and a greater relative body fat, particularly the truncal fat accumulation which may in turn lead to insulin resistance and adverse metabolism (24). It is possible that an unfavorable intrauterine environment may generate the adaptation of adipose tissue's function and distribution to favor MONW. However, the mechanisms underlying this adaptation warrant further investigation.

To date, limited data exist with regard to genetic predisposition to MONW (25). Although a limited number of genetic variants (20 SNPs) were examined, our study presents the first convincing evidence that CDKAL1 rs2206734 is associated with protection from the MONW phenotype. This is consistent with previous reports of the $\mathrm{C}$ allele of CDKAL1 rs2206734 being associated with higher BMI but reduced risk of T2D in East Asian adults (26). Similarly, recent studies have identified common genetic variants, including that near insulin receptor substrate 1 (IRS1), where specific alleles were associated with elevated BMI and body fat percentage, but reduced cardiometabolic risk $(4,27,28)$. Given the well-established association between obesity and cardiovascular risk, these results seem counterintuitive. However, these 'high BMI alleles' have all been associated with disproportionate s.c.to-visceral adipose tissue ratios, gynoid fat distribution, and greater insulin sensitivity $(4,28)$. These loci may affect metabolic health by regulating the capacity of s.c. adipose tissue to store excess lipid and support the 'adipose tissue expandability' hypothesis which states that when adipose tissue is unable to undergo healthy hyperplasia and hypertrophy to cope with an increased demand for lipid storage, the excess lipid accumulates in the visceral compartment, thus predisposing to insulin resistance and cardiometabolic risk. It is interesting to note that CDKAL1 is a methylthiotransferase that enhances translational efficiency and is broadly expressed in metabolic tissues, including adipose tissue and pancreatic $\beta$ cells $(29,30)$. Thus, CDKAL1 rs2206734 may protect against MONW through pathways of adipose tissue expansion or $\beta$ cell function by influencing translation, given that the MONW protective $C$ allele was also associated with lower TG $(P=0.011)$ and glucose levels $(P=0.005)$ in our study (Supplementary Table 2 ). In contrast to a previous study of 62,245 East Asian adults (26), but consistent with a study in children from Greece (31), the observed relationship between rs2206734 and metabolic disorders was not accompanied by an association with BMI in our children, although we had more than $80 \%$ power to detect a per-allele effect on BMI of 0.035 and $0.039 \mathrm{~kg} / \mathrm{m}^{2}$ with allele frequency of $43 \%$ in an additive model. This contradiction may result from the genetic association with body fat being less penetrant in children. In this case, the associated mechanisms in children warrant further study.

Another novel and important finding of our study is that the genetic association of CDKAL1 rs2206734 with the MONW phenotype was altered by birthweight. Given that another locus at $C D K A L 1$, i.e. rs7756992, has been recognized as a potential link between low birthweight and T2D according to the fetal insulin hypothesis $(32,33)$, we speculate that the significant association between CDKAL1 rs2206734 and the MONW phenotype might be due to its association with birthweight, although these two variants are not in perfect linkage disequilibrium with one another $\left(r_{\mathrm{CHB}}^{2}=0.620\right)$. However, there was no direct association between CDKAL1 rs2206734 and birthweight in our study; but rather, an interaction between the rs2206734 locus and birthweight was discovered. Interestingly, a stratified analysis according to birthweight categories revealed that the protective association between the rs2206734 locus and MONW was most pronounced in children with high birthweight, while this association was lost in children with low and intermediate birthweight. This suggests that exposure to an adverse intrauterine environment may contribute to the loss of the protective cardiometabolic effect at this locus, whereas maternal and/or placental factors which increase birthweight may be necessary for this gene variant to exert its cardio-protective effects.

In addition to the effects beginning in utero, the effects of environmental exposures on gene expression were perceived to extend through childhood development, but the mechanisms responsible for these complex interactions between genetic and environmental factors remain unclear (34). Our study revealed that the effect of high birthweight to strengthen the protective influence of 
the CDKAL1 locus on MONW appears to be augmented by a favorable childhood (postnatal) environment, whereas an adverse childhood environment could weaken the protective effect at CDKAL1 by more than 50\%. Given that CDKAL1 is broadly expressed in adipose tissue, the possibility that epigenetic regulation of adipose tissue function during the adaptation to intrauterine and childhood environments may be involved in these geneenvironment interactions warrants further investigation. Nonetheless, our study demonstrates that modification of the intrauterine and early childhood environments has important implications for prevention of MONW, particularly in genetically susceptible individuals.

Our study can claim several strengths. The principal strength of our analysis was the availability of a large, wellcharacterized cohort of Chinese children as it afforded an opportunity to examine not only the participants but also the combined effects of numerous environmental factors. The collective examination of genetic and environmental factors (as well as the interactions thereof) is another strength. Notwithstanding, several limitations in our study should be noted. Firstly, our examination encompassed only a limited number of genetic variants associated with adult BMI or glucose metabolism. Our novel finding that the BMI-related allele in CDKAL1 showed a protective association against MONW, along with findings from recent studies in adults $(4,25)$, suggests that future studies in children should focus on genes relevant to adipose tissue expandability and storage. Secondly, the lack of consensus on the definition of MONW may result in difficulty comparing our results with those of previous and subsequent pediatric studies. Thirdly, because Asians tend to have cardiometabolic abnormalities at relatively low levels of BMI, our study may have more significant public health implications in Asian populations than other ethnicities. Finally, due to the cross-sectional design of our study, it is not possible to establish causality. Our ongoing prospective study will more definitively elucidate the role of these various factors in the development and persistence of MONW from childhood onward.

In conclusion, our study demonstrates that early environmental exposures, including lifestyle and socioeconomic factors during childhood, as well as birthweight, play important roles in determining the MONW phenotype. Our findings furthermore suggest that the protective CDKAL1 rs2206734 allele may be a novel and promising locus for future research into the genetic origins of cardiovascular disease, and a deeper understanding of the MONW phenotype in particular. Specifically, the observation that the genetic protection at the CDKAL1 locus could be mitigated by birthweight suggests the possibility of an epigenetic mechanism in determining the MONW phenotype. Furthermore, our finding that favorable socioeconomic status could strengthen this genetically determined protective effect suggests that environmental modifications may be disproportionately effective at preventing cardiovascular disease in individuals with the protective CDKAL1 rs2206734 allele. Given that the prevention and detection of metabolic abnormalities is largely unnoticed in normal-weight individuals until the onset of a chronic diseases like T2D, our study provides new insights into potential prevention strategies for the MONW phenotype, especially in genetically susceptible individuals. Future work is needed to elucidate the genetic and epigenetic mechanisms at work to determine the increased risk of cardiovascular disease in metabolically obese, normal weight individuals.

\section{Supplementary materials}

This is linked to the online version of the paper at https://doi.org/10.1530/ EJE-19-0755.

\section{Declaration of interest}

The authors declare that there is no conflict of interest that could be perceived as prejudicing the impartiality of this study.

\section{Funding}

This work was supported by grants from Beijing Natural Science Foundation (\#7172169), National Key R\&D Program of China (\#2016YFC1304801), Beijing Municipal Science and Technology Commission (\#D111100000611001 and \#D111100000611002); National Key Program of Clinical Science of China (\#WBYZ2011-873); Novo Nordisk Union Diabetes Research Talent Fund (\#2011A002), the Non-profit Central Research Institute Fund of Chinese Academy of Medical Sciences (\#2017PT32020 and \#2018PT32001); Beijing Science \& Technology Star Program (\#2004A027) and Jingxi Scientific Program of Beijing Chaoyang Hospital (\#JXPY201606).

\section{Author contribution statement}

$\mathrm{M} \mathrm{Li}$, J Mi and S Gao contributed equally to this study.

\section{Acknowledgments}

The authors thank BCAMS participants for their continuing participation in this research effort.

\section{References}

1 Stefan N, Schick F \& Haring HU. Causes, characteristics, and consequences of metabolically unhealthy normal weight in humans. Cell Metabolism 201726 292-300. (https://doi.org/10.1016/j. cmet.2017.07.008) 
2 Razak F, Anand SS, Shannon H, Vuksan V, Davis B, Jacobs R, Teo KK, McQueen M \& Yusuf S. Defining obesity cut points in a multiethnic population. Circulation 2007115 2111-2118. (https:// doi.org/10.1161/CIRCULATIONAHA.106.635011)

3 Chiu M, Austin PC, Manuel DG, Shah BR \& Tu JV. Deriving ethnicspecific BMI cutoff points for assessing diabetes risk. Diabetes Care 201134 1741-1748. (https://doi.org/10.2337/dc10-2300)

4 Lotta LA, Gulati P, Day FR, Payne F, Ongen H, van de Bunt M, Gaulton KJ, Eicher JD, Sharp SJ, Luan J et al. Integrative genomic analysis implicates limited peripheral adipose storage capacity in the pathogenesis of human insulin resistance. Nature Genetics 201749 17-26. (https://doi.org/10.1038/ng.3714)

5 Neeland IJ, Poirier P \& Despres JP. Cardiovascular and metabolic heterogeneity of obesity: clinical challenges and implications for management. Circulation 2018137 1391-1406. (https://doi. org/10.1161/CIRCULATIONAHA.117.029617)

6 Wen W, Zheng W, Okada Y, Takeuchi F, Tabara Y, Hwang JY, Dorajoo R, Li H, Tsai FJ, Yang X et al. Meta-analysis of genome-wide association studies in East Asian-ancestry populations identifies four new loci for body mass index. Human Molecular Genetics 201423 5492-5504. (https://doi.org/10.1093/hmg/ddu248)

7 Cho YS, Chen CH, Hu C, Long J, Ong RT, Sim X, Takeuchi F, Wu Y, Go MJ, Yamauchi T et al. Meta-analysis of genome-wide association studies identifies eight new loci for type 2 diabetes in east Asians. Nature Genetics 201144 67-72. (https://doi.org/10.1038/ng.1019) 8 Liu J, Wang L, Qian Y, Dai J, Shen C, Jin G, Hu Z \& Shen H. Association of 48 type 2 diabetes susceptibility loci with fasting plasma glucose and lipid levels in Chinese Hans. Diabetes Research and Clinical Practice 2018139 114-121. (https://doi.org/10.1016/j. diabres.2018.02.039)

9 Prince RL, Kuk JL, Ambler KA, Dhaliwal J \& Ball GD. Predictors of metabolically healthy obesity in children. Diabetes Care 201437 1462-1468. (https://doi.org/10.2337/dc13-1697)

10 Phillips CM. Metabolically healthy obesity across the life course: epidemiology, determinants, and implications. Annals of the New York Academy of Sciences 20171391 85-100. (https://doi.org/10.1111/ nyas.13230)

11 Schreier HM \& Chen E. Socioeconomic status and the health of youth: a multilevel, multidomain approach to conceptualizing pathways. Psychological Bulletin 2013139 606-654. (https://doi. org/10.1037/a0029416)

12 Wahlqvist ML, Krawetz SA, Rizzo NS, Dominguez-Bello MG, Szymanski LM, Barkin S, Yatkine A, Waterland RA, Mennella JA, Desai M et al. Early-life influences on obesity: from preconception to adolescence. Annals of the New York Academy of Sciences 20151347 1-28. (https://doi.org/10.1111/nyas.12778)

13 Li L, Yin J, Cheng H, Wang Y, Gao S, Li M, Grant SF, Li C, Mi J \& $\mathrm{Li} \mathrm{M}$. Identification of genetic and environmental factors predicting metabolically healthy obesity in children: data From the BCAMS study. Journal of Clinical Endocrinology and Metabolism 2016101 1816-1825. (https://doi.org/10.1210/jc.2015-3760)

14 Wang Q, Yin J, Xu L, Cheng H, Zhao X, Xiang H, Lam HS, Mi J \& Li M. Prevalence of metabolic syndrome in a cohort of Chinese schoolchildren: comparison of two definitions and assessment of adipokines as components by factor analysis. BMC Public Health 2013 13 249. (https://doi.org/10.1186/1471-2458-13-249)

15 Li G, Yin J, Fu J, Li L, Grant SFA, Li C, Li M, Mi J, Li M \& Gao S. FGF21 deficiency is associated with childhood obesity, insulin resistance and hypoadiponectinaemia: the BCAMS Study. Diabetes and Metabolism 201743 253-260. (https://doi.org/10.1016/j. diabet.2016.12.003)

16 Matthews DR, Hosker JP, Rudenski AS, Naylor BA, Treacher DF \& Turner RC. Homeostasis model assessment: insulin resistance and beta-cell function from fasting plasma glucose and insulin concentrations in man. Diabetologia 198528 412-419. (https://doi. org/10.1007/bf00280883)

17 Gabriel S, Ziaugra L \& Tabbaa D. SNP genotyping using the Sequenom MassARRAY iPLEX platform. Current Protocols in Human Genetics 2009 Chapter 2 Unit 2.12. (https://doi. org/10.1002/0471142905.hg0212s60)

18 Kuzik N, Carson V, Andersen LB, Sardinha LB, Grontved A, Hansen BH, Ekelund U \& International Children's Accelerometry Database. Physical activity and sedentary time associations with metabolic health across weight statuses in children and adolescents. Obesity 201725 1762-1769. (https://doi.org/10.1002/oby.21952)

19 Darmon N \& Drewnowski A. Does social class predict diet quality? American Journal of Clinical Nutrition 200887 1107-1117. (https:// doi.org/10.1093/ajen/87.5.1107)

20 Giles-Corti B \& Donovan RJ. Socioeconomic status differences in recreational physical activity levels and real and perceived access to a supportive physical environment. Preventive Medicine 200235 601-611. (https://doi.org/10.1006/pmed.2002.1115)

21 Barker DJ, Gluckman PD, Godfrey KM, Harding JE, Owens JA \& Robinson JS. Fetal nutrition and cardiovascular disease in adult life. Lancet 1993341 938-941. (https://doi.org/10.1016/01406736(93)91224-a)

22 Lillycrop KA \& Burdge GC. Environmental challenge, epigenetic plasticity and the induction of altered phenotypes in mammals. Epigenomics 20146 623-636. (https://doi.org/10.2217/epi.14.51)

23 Bouhours-Nouet N, Dufresne S, de Casson FB, Mathieu E, Douay O, Gatelais F, Rouleau S \& Coutant R. High birth weight and early postnatal weight gain protect obese children and adolescents from truncal adiposity and insulin resistance: metabolically healthy but obese subjects? Diabetes Care 200831 1031-1036. (https://doi. org/10.2337/dc07-1647)

24 Gale CR, Martyn CN, Kellingray S, Eastell R \& Cooper C. Intrauterine programming of adult body composition. Journal of Clinical Endocrinology and Metabolism 200186 267-272. (https://doi. org/10.1210/jcem.86.1.7155)

25 Loos RJ. Integrating publicly available genome-wide association data to study the genetic basis of metabolically healthy obese and metabolically obese but normal-weight individuals. Diabetes 201463 4004-4007. (https://doi.org/10.2337/db14-1358)

26 Okada Y, Kubo M, Ohmiya H, Takahashi A, Kumasaka N, Hosono N, Maeda S, Wen W, Dorajoo R, Go MJ et al. Common variants at CDKAL1 and KLF9 are associated with body mass index in east Asian populations. Nature Genetics 201244 302-306. (https://doi. org/10.1038/ng.1086)

27 Yaghootkar H, Lotta LA, Tyrrell J, Smit RA, Jones SE, Donnelly L, Beaumont R, Campbell A, Tuke MA, Hayward C et al. Genetic evidence for a link between favorable adiposity and lower risk of Type 2 diabetes, hypertension, and heart disease. Diabetes 201665 2448-2460. (https://doi.org/10.2337/db15-1671)

28 Scott RA, Fall T, Pasko D, Barker A, Sharp SJ, Arriola L, Balkau B, Barricarte A, Barroso I, Boeing $\mathrm{H}$ et al. Common genetic variants highlight the role of insulin resistance and body fat distribution in type 2 diabetes, independent of obesity. Diabetes 201463 4378-4387. (https://doi.org/10.2337/db14-0319)

29 Wei FY, Suzuki T, Watanabe S, Kimura S, Kaitsuka T, Fujimura A, Matsui H, Atta M, Michiue H, Fontecave M et al. Deficit of tRNA(Lys) modification by Cdkal1 causes the development of type 2 diabetes in mice. Journal of Clinical Investigation 2011121 3598-3608. (https:// doi.org/10.1172/JCI58056)

30 Palmer CJ, Bruckner RJ, Paulo JA, Kazak L, Long JZ, Mina AI, Deng Z, LeClair KB, Hall JA, Hong S et al. Cdkal1, a type 2 diabetes susceptibility gene, regulates mitochondrial function in adipose tissue. Molecular Metabolism 20176 1212-1225. (https://doi. org/10.1016/j.molmet.2017.07.013) 
31 Rask-Andersen M, Philippot G, Moschonis G, Dedoussis G, Manios Y, Marcus C, Fredriksson R \& Schioth HB. CDKAL1-related single nucleotide polymorphisms are associated with insulin resistance in a cross-sectional cohort of Greek children. PLoS ONE 20149 e93193. (https://doi.org/10.1371/journal.pone.0093193)

32 Zhao J, Li M, Bradfield JP, Wang K, Zhang H, Sleiman P, Kim CE, Annaiah K, Glaberson W, Glessner JT et al. Examination of type 2 diabetes loci implicates CDKAL1 as a birth weight gene. Diabetes 200958 2414-2418. (https://doi.org/10.2337/db09-0506)
33 Andersson EA, Pilgaard K, Pisinger C, Harder MN, Grarup N, Faerch K, Poulsen P, Witte DR, Jorgensen T, Vaag A et al. Type 2 diabetes risk alleles near ADCY5, CDKAL1 and HHEX-IDE are associated with reduced birthweight. Diabetologia 201053 1908-1916. (https://doi.org/10.1007/s00125-010-1790-0)

34 Hochberg Z, Feil R, Constancia M, Fraga M, Junien C, Carel JC, Boileau P, Le Bouc Y, Deal CL, Lillycrop K et al. Child health, developmental plasticity, and epigenetic programming. Endocrine Reviews 201132 159-224. (https://doi.org/10.1210/er.2009-0039)

Received 23 September 2019

Revised version received 16 January 2020

Accepted 29 January 2020 